\title{
Metal ion quenching of perylene fluorescence in lipid bilayers
}

\author{
D J S Birch, K Suhling, A S Holmes, T Salthammer ${ }^{+}$and R E Imhof \\ Department of Physics and Applied Physics, University of Strathclyde, Glasgow, UK \\ +Present address: WKI-Fraunhofer-Arbeitsgruppe für Holzforschuing, Bienrader \\ WEG 54E, 3300 Braunschweig, Germany
}

\begin{abstract}
Perylene fluorescence was found to be quenched by $\mathrm{Co}^{2+}$ and $\mathrm{Ni}^{2+}$ ions below the phase transition in small unilamellar vesicles. Long-range Förster dipole-dipole energy transfer was found to describe the quenching of the perylene fluorescence in the case of $\mathrm{Co}^{2+}$ whereas for $\mathrm{Ni}^{2+}$ the quenching mechanism was found to be more akin to the shorter range Dexter exchange interaction. $\mathrm{A} \mathrm{Co}^{2+}$ sensor in solution based on Förster energy transfer from perylene is proposed.
\end{abstract}

\section{INTRODUCTION}

Fluorescence probe techniques are now well established in the study of membranes and other ordered media ${ }^{[1]}$. The quenching of aromatic fluorescence by metal ions can involve a number of mechanisms including electron transfer, Dexter exchange, heavy atom induced inter-system crossing and Forster energy transfer. Energy transfer studies in ordered media are singularly useful in describing the locations of both donors and acceptors, which are usually both aromatic molecules. An alternative approach, which we have been developing and will report here, is to use transition metal ions as acceptors $[2,3]$.

Many of the transition metal ions like $\mathrm{Co}^{2+}$ and $\mathrm{Ni}^{2+}$ are useful in the context of non-radiative energy transfer as their absorption spectra in solution show good overlap with many of the widely used aromatic probes. The lack of radiative emission from such ions is a further advantage over using aromatic acceptors. In aqueous solution cobalt and nickel exist as the hexaaquo ions $\left[\mathrm{Co}_{0} 6 \mathrm{H}_{2} \mathrm{O}\right]^{2+}$ and $\left[\mathrm{Ni}^{2} 6 \mathrm{H}_{2} \mathrm{O}\right]^{2+}$. Therefore in membranes we would expect these to be bound to the anionic lipid headgroup. Such site-specific location is a further useful property of metal ion acceptors. As a non-polar, nearly disk-shaped molecule perylene is thought to lie within the hydrocarbon region of a bilayer. The fluorescence properties of perylene are well documented both in homogeneous solutions ${ }^{[4]}$ and lipid bilayers ${ }^{[5]}$. In summary, the perylene-transition metal ion interaction is a relatively well defined problem.

Recently we have reported the quenching of perylene fluorescence by $\mathrm{Co}^{2+}$ ions in glycerol as being well described by Förster long-range dipole-dipole energy transfer with an interaction radius of $13.2 \AA^{[6]}$. However, in lipid bilayers a complication arises in the kinetics due to perylene-perylene dipole-dipole energy transfer as a consequence of clustering of the perylene molecules ${ }^{[7]}$. In this paper we compare and contrast the quenching mechanisms for cobalt and nickel ions in glycerol and in small unilamellar vesicles of $L-\alpha-$ dipalmitoylphosphatidylcholine (DPPC).

Our long term objectives in this area are to obtain a better understanding of the kinetics involved in the fluorescence quenching of aromatic molecules by metal ions in ordered media and hence develop metal ion sensors using fluorescence energy transfer.

\section{EXPERIMENTAL}

Perylene (Aldrich Gold Label) was used as received. $\mathrm{CoCl}_{2} .6 \mathrm{H}_{2} \mathrm{O}$ (Merck p.a.) and $\mathrm{NiCl}_{2} .6 \mathrm{H}_{2} \mathrm{O}$ were recrystallized from methanol. L- $\alpha$-dipalmitoylphosphatidylcholine (DPPC) was obtained from the Sigma Chemical Co.

Preparation of the small unilammelar vesicles (SUV) was described previously[7].

Fluorescence spectra were recorded with either a Perkin-Elmer MPF-44 E spectrofluorimeter with a quantum correction unit and thermostated cuvette holder or a Shimadzu RF-540 spectrofluorimeter. All fluorescence spectra were corrected for reabsorption of the hydrated ion complexes. 
Fluorescence decay kinetics were measured using the technique of time-correlated single photon counting. Either the coaxial flashlamp filled with hydrogen[8] or a Hamamatsu PLP-01 frequency doubled diode laser operated at $10 \mathrm{MHz}$ repetition rate was used for excitation. Operating the lamp at $40 \mathrm{kHz}$ gives an instrumental pulse $\sim 1.5 \mathrm{~ns}$ fwhm. The diode laser gave an instrumental pulse width of $\sim 350 \mathrm{ps}$ fwhm. The excitation wavelength for all measurements was $385 \mathrm{~nm}$. Prism polarisers were used at the "magic angle" to eliminate rotational effects. The sample temperature was controlled by electrical heating (Eurotherm) with a precision of $\pm 0.5 \mathrm{~K}$. The maximum number of counts in a channel was between $1.10^{4}$ and $2.10^{4}$ recorded at channel widths down to $84 \mathrm{ps} / \mathrm{ch}$. Data analysis was performed using the IBH iterative reconvolution library.

\section{THEORY}

The most widely encountered model for describing non-radiative energy transfer is that originally described by Förster for dipole-dipole interactions ${ }^{[1,9]}$. The original expression derived by Förster for energy transfer predicts a fluorescence response of the form

$$
I(t)=I_{0} \exp \left[-\frac{t}{\tau_{0}}-2 \gamma\left(\frac{t}{\tau_{0}}\right)^{1 / 2}\right]
$$

where $\tau_{0}$ is the unquenched lifetime of the donor and $\gamma=[\mathrm{A}] / \mathrm{C}_{\mathrm{AO}} . \quad[\mathrm{A}]$ is the concentration of acceptor and $\mathrm{C}_{\mathrm{AO}}$ is the critical acceptor concentration for energy transfer. This parameter is related to the critical transfer distance $R_{0}$ via

$$
\mathrm{C}_{\mathrm{AO}}=\frac{3}{2 \pi^{3 / 2} \mathrm{~N}_{\mathrm{A}} \mathrm{R}_{0}^{3}}
$$

$\mathbf{R}_{\mathbf{o}}$ can also be determined theoretically from the overlap of the donor emission spectrum and the acceptor absorption spectrum by the equation

$$
R_{0}^{6}=\frac{9 \ln (10) \kappa^{2} \phi_{\mathrm{o}}}{128 \pi^{3} \mathrm{~N}_{\mathrm{A}} n^{4}} \times \int_{0}^{\infty} \frac{F_{\mathrm{d}}(\tilde{v}) \varepsilon_{\mathrm{a}}(\tilde{v})}{\tilde{v}^{4}} \mathrm{~d} \tilde{v}
$$

where $\phi_{o}$ is the emission quantum yield of the donor in the absence of the quencher, $\kappa$ is the orientation factor $\left(K^{2}=0.67\right.$ for randomly distributed molecules) and $n$ is the refractive index of the solvent, $F_{d}(v)$ is the emission intensity of the donor and $\varepsilon_{\mathrm{a}}(v)$ the extinction coefficient of the acceptor at the wavenumber $v$.

The relative donor quantum yield in Förster energy transfer can be expressed as ${ }^{[1]}$

$$
\frac{\phi}{\phi_{0}}=1-\pi^{1 / 2} \gamma \exp \left(\gamma^{2}\right) \operatorname{erfc}(\gamma)
$$

where erfc is the complementary error function. At small quencher concentrations Eqn (4) reduces to

$$
\frac{\phi}{\phi_{0}}=1-\pi^{1 / 2} \gamma
$$

In the case where dipole-dipole interaction from the donor-donor transfer is comparable to the donor-acceptor transfer, the donor fluorescence decay can be described by a development of the simple Förster model as described by Huber ${ }^{[10]}$ :

$$
I(t)=I_{0} \exp \left[-\frac{t}{\tau_{0}}-\left(\sqrt{2} \gamma_{D}+2 \gamma_{A}\right)\left(\frac{t}{\tau_{0}}\right)^{1 / 2}\right]
$$

where the subscripts D and A refer to the donor and acceptor respectively. The reduced factor for the donor transfer takes into account the reversible migration of energy among the donor molecules, contrary to the irreversible transfer from donors to acceptors.

At shorter ranges of interaction (ie $<<50 \AA$ ) than that over which Förster energy transfer is dominant electron exchange interactions and higher multipole-multipole interactions can become dominant, particularly when dipole-dipole interactions are forbidden. The principle requirement for such so called Dexter electron exchange is overlap of the excited donor and acceptor molecular orbitals. A specific feature of the Dexter 
mechanism is that unlike the Forster mechanism the energy transfer rate is independent of oscillator strength of the acceptor transition, which in the case of $\mathrm{Ni}^{2+}$, is $\sim 20$ times less than for $\mathrm{Co}^{2+[11]}$.

Distinguishing between Förster and Dexter energy transfer is often not readily achieveable at short distances of interaction and has been little explored by comparing the fit of the fluorescence decay to the appropriate impulse responses. In contrast to Eqn (4) describing Förster kinetics the equivalent expression for Dexter kinetics is of the form[11]

$$
\frac{\phi}{\phi_{0}}=\exp \left[-\frac{[\mathrm{A}]}{\mathrm{C}_{\mathrm{AO}}}\right]
$$

Hence the shape of $\phi / \phi_{o}$ vs [A] provides, in theory at least, one method of distinguishing between the two. In addition it can be shown that the quantity $\rho$, as given in Eqn (8), has a value of $\sim 0.5$ for dipole - dipole interactions and a value of $\sim 0.1$ for exchange interaction ${ }^{[11]}$.

$$
\rho=\lim _{[\mathrm{A}] \rightarrow 0} \frac{d\left(\tau / \tau_{0}\right) / d[\mathrm{~A}]}{d\left(\phi / \phi_{0}\right) / d[\mathrm{~A}]}
$$

We can now discuss the applicability of these various models to the behaviour of the perylene-metal ion interactions which we have observed below the phase transition in DPPC bilayers (ie in the gel phase) where the effects of diffusion are minimal.

\section{RESULTS AND DISCUSSION}

The excitation and fluorescence spectra for perylene in DPPC bilayers and the absorption spectrum for $\mathrm{Co}^{2+}$ and $\mathrm{Ni}^{2+}$ ions bound to the lipid headgroup at $\mathrm{T}=30 \mathrm{C}$ are shown in Fig 1. These spectra are similar to those found in alcoholic solution, hence energy transfer is the expected quenching mechanism ${ }^{[6]}$. The $\mathrm{Co}^{2+}$ and $\mathrm{Ni}^{2+}$ absorption spectra can be seen to be on the low and high energy side of the perylene emission spectra respectively. In the case of $\mathrm{Ni}^{2+}$ significantly more overlap with the perylene absorption is also evident.

We measured the fluorescence lifetime of perylene in DPPC bilayers in the gel phase to be $6.31 \mathrm{~ns}$. This is in agreement with that found by others in membranes ${ }^{[5]}$. However, we obtained a $\chi^{2}$ value of 1.31 and nonrandom residuals which suggested that the decay was non-exponential.

\section{PERYLENE-COBALT STUDIES}

On the addition of cobalt chloride to the liposome, a monoexponential fit to the perylene fluorescence decay is inappropriate in all cases. Table 1 gives the kinetic parameters obtained when analysing the data using Förster's model for energy transfer (Eqn 1) which gives a much improved fit. The results given here are for 3D Förster analysis which has previously been shown to give slightly better fits than $2 \mathrm{D}$ analysis ${ }^{[12]}$. The nearly constant value for $\tau_{0}$ obtained in the analysis suggests that Förster type energy transfer is indeed taking place and diffusion quenching is negligible. However, when the interaction radius, $R_{0}$, is calculated from Eqn (2) the values obtained for $R_{0}$ are not constant as expected but decrease with increasing $\mathrm{Co}^{2+}$ concentration. From Eqn (3) $R_{o}$ is expected to be $\approx 12.4 \AA$ and $\approx 13.2 \AA$ from time-resolved studies in glycerol solution[6]. Hence $R_{o}$ in the ordered media at cobalt concentrations $\geq 0.06 \mathrm{~mol} \mathrm{l}^{-1}$ (ie cobalt:lipid ratios $>75: 1$ ) are in good agreement with the expected value. However, at the low acceptor concentrations the values obtained for $R_{0}$ are too high.

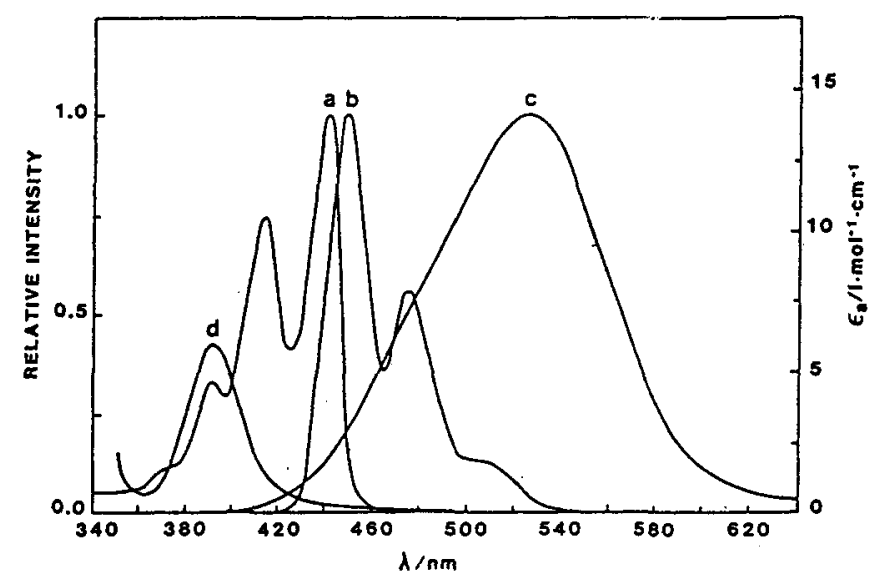

Fig 1 Excitation (a) and fluorescence (b) spectra for perylene in lipid bilayers and the absorption spectra for $\mathrm{Co}^{2+}$ (c) and $\mathrm{Ni}^{2+}$ (d) ions bound to the lipid headgroup at $\mathrm{T}=30 \mathrm{C}$. 
Table 1 Kinetic parameters obtained from analysis using Förster kinetics and also values for $\gamma_{\mathrm{A}}$ and $\mathrm{R}_{\mathrm{o}}$ taking donor-energy transfer into consideration for the fluorescence quenching of perylene by $\mathrm{Co}^{2+}$ ions in DPPC vesicles at $30^{\circ} \mathrm{C}$ and a lipid to probe ratio of 200:1

\begin{tabular}{|c|c|c|c|c|c|c|}
\hline \multirow[t]{2}{*}{$\mathrm{Co}^{2+}\left(\mathrm{mol} \mathrm{l}^{-1}\right)$} & \multicolumn{4}{|c|}{ Förster kinetics } & \multirow{2}{*}{$\gamma_{A}{ }^{b)}$} & \multirow{2}{*}{$\left.\mathrm{R}_{\mathrm{o}} \mathrm{c}\right)(\AA)$} \\
\hline & $\tau_{\mathrm{o}}(\mathrm{ns})$ & $\gamma$ & $\chi^{2}$ & $\left.R_{0}{ }^{a}\right)(\AA)$ & & \\
\hline $\begin{array}{l}0.011 \\
0.021 \\
0.031 \\
0.041 \\
0.060 \\
0.080 \\
0.100\end{array}$ & $\begin{array}{l}6.60 \pm 0.21 \\
6.45 \pm 0.21 \\
6.38 \pm 0.24 \\
6.37 \pm 0.24 \\
5.95 \pm 0.24 \\
5.96 \pm 0.27 \\
6.15 \pm 0.30\end{array}$ & $\begin{array}{l}0.138 \\
0.208 \\
0.299 \\
0.352 \\
0.392 \\
0.430 \\
0.529\end{array}$ & $\begin{array}{l}1.25 \\
1.05 \\
1.22 \\
1.13 \\
1.18 \\
1.23 \\
1.30\end{array}$ & $\begin{array}{l}17.7 \pm 0.3 \\
16.4 \pm 0.2 \\
16.3 \pm 0.3 \\
15.7 \pm 0.1 \\
14.3 \pm 0.1 \\
13.4 \pm 0.1 \\
13.3 \pm 0.1\end{array}$ & $\begin{array}{l}0.053 \\
0.123 \\
0.214 \\
0.267 \\
0.307 \\
0.345 \\
0.444\end{array}$ & $\begin{array}{l}12.9 \pm 1.0 \\
13.8 \pm 0.5 \\
14.6 \pm 0.3 \\
14.3 \pm 0.3 \\
13.2 \pm 0.2 \\
12.4 \pm 0.2 \\
12.6 \pm 0.2\end{array}$ \\
\hline
\end{tabular}

a) Calculated from Eqns (1) and (2) b) from Eqn (6) c) from Eqns (2) and (6).

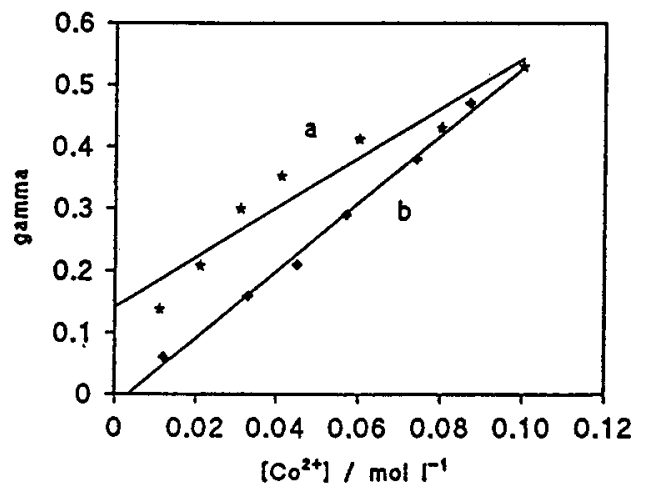

Fig 2 Plot of $\gamma$ versus $\left[\mathrm{Co}^{2+}\right]$ for perylenecobalt energy transfer and (a) lipid bilayers (b) glycerol

The interaction radius can also be obtained from the gradient of the graph of $\gamma$ versus $\left[\mathrm{Co}^{2+}\right]$ which is shown in Fig 2 for the lipid bilayer. This gives a value for $R_{0}$ of $12.1 \pm 1.6 \AA$ which agrees within the error with the expected value. However, this plot should go through zero as expected from Eqn (2) and observed for perylene-cobalt energy transfer in glycerol (see fig. 2). This suggests that there is an additional energy transfer process occurring in the lipid bilayer which is predominant at the low acceptor concentrations. We can account for the anomalies seen here by radiationless energy transfer between the perylene molecules. On analysing the perylene in the lipid data with no quencher added, using Forster kinetics a reduced $\chi^{2}$ of 1.06 was obtained and a $\gamma$ value of $0.120 \pm 0.006$, which is close, given the error, to the intercept of the straight-line graph shown in Fig 2 for perylene/cobalt quenching in a bilayer.

Given that the energy transfer rate for perylene-perylene is comparable to that for perylene-cobalt at the low acceptor concentrations we can analyse the results using Eqn (6). Taking the experimental value for $\gamma_{D}$ of 0.120 we can calculate a corrected value of $\gamma_{\mathrm{A}}$ via the equation $\gamma_{\mathrm{A}}=\gamma-\gamma_{\mathrm{D}} / \sqrt{2}$ where the $\gamma$ values are taken from Table 1. The $\gamma_{A}$ values are also given in Table 1 as well as the corrected values for $R_{0}$ which are now constant with a mean value of $13.4 \dot{A}$, in good agreement with the theoretical value and that found in glycerol.

In addition to the evidence for donor-donor energy transfer due to perylene clustering already presented, a study of perylene in DPPC vesicles, in the absence of cobalt quenching, showed insignificant change in $\gamma$ over two orders of magnitude change lipid to probe ratio[7]. This means that the perylene molecules cluster to a local concentration independent of the macroscopic lipid to probe ratio. Figure 3 shows a good Förster fit to perylene in DPPC measured using diode laser excitation even at a lipid to probe ratio as low as 3700:1. Using the overlap of the perylene absorption and fluorescence spectra Eqn (3) predicts $R_{0}=35.7 \AA$ for perylene.

\section{PERYLENE-NICKEL STUDIES}

Judging from the $\chi^{2}$ values alone Table 2 indicates good fits to the 2-D Förster model for nickel quenching also. However, a number of discrepancies with both the predictions of the Förster model and the $\mathrm{Co}^{2+}$ results shown in Table 1 are immediately evident. In particular $\tau_{\mathrm{o}}$ for $\mathrm{Ni}^{2+}$ quenching is anything but constant and systematically decreases with increasing $\left[\mathrm{Ni}^{2+}\right]$. Because of the low fluidity of the gel phase it is extremely 


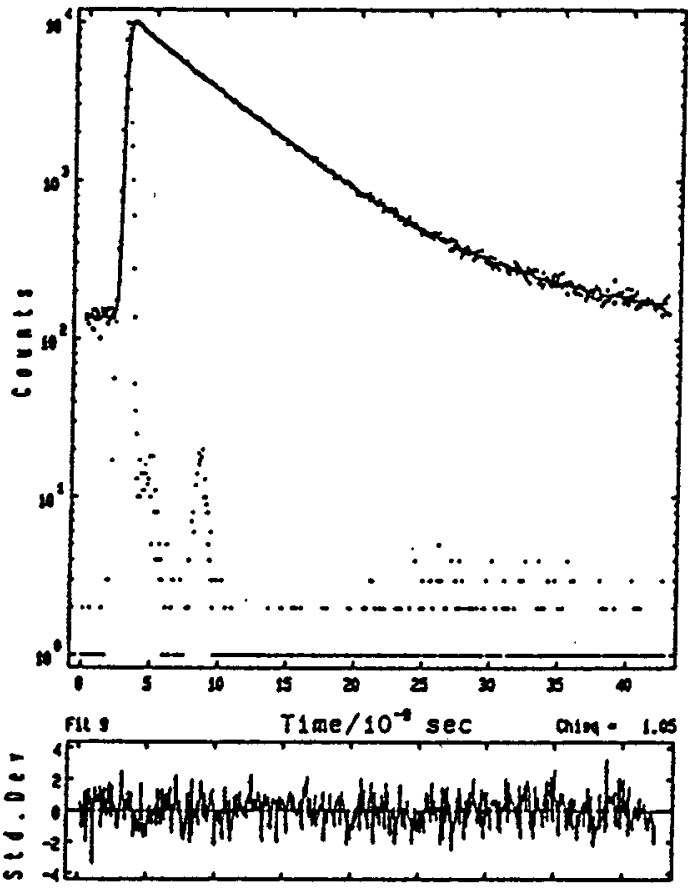

Fig 3 3D Förster fit to 3700:1 DPPC:perylene at $30^{\circ} \mathrm{C}$ measured using diode laser excitation in $1 \frac{1}{2}$ hours. Front face excitation and detection were used. This fit gave $\tau_{0}=7.14 \mathrm{~ns}$ and $\gamma=0.130$

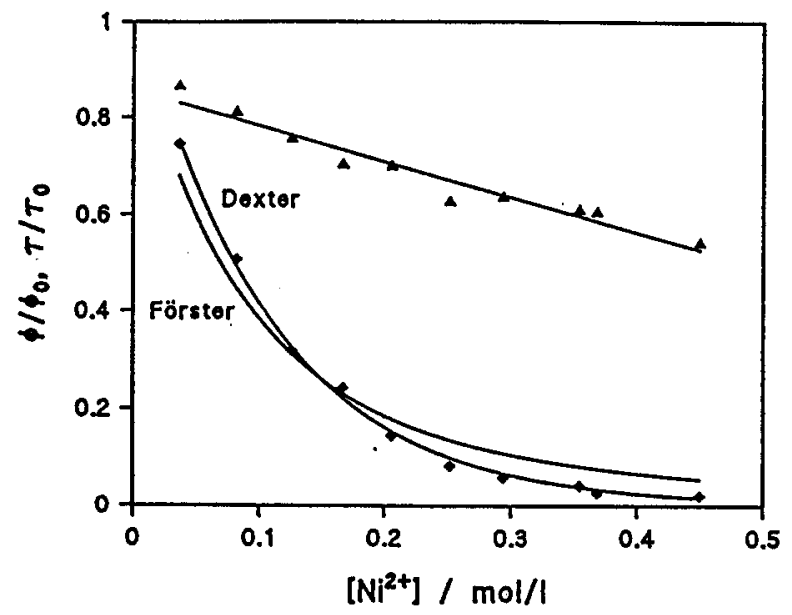

Fig 4 Stern-Volmer plots for perylene fluorescence quenched by $\mathrm{Ni}^{2+}$ in glycerol at $25^{\circ} \mathrm{C} . \phi / \phi_{0}$ is fitted to both Förster and Dexter mechanisms.

unlikely that this is due to collisional quenching. In addition, for $\mathrm{Ni}^{2+}$ quenching the $2 \mathrm{D}$ Förster model gives a slight improvement over the 3D model although there was little difference in the $\tau_{0}$ and $\gamma$ values, whereas with $\mathrm{Co}^{2+}$ the opposite was true ${ }^{[12]}$.

The Stern-Volmer plot of $\phi_{0} / \phi$ for perylene in DPPC at $30 \mathrm{C}$ quenched by $\mathrm{Ni}^{2}$ shows a slight positive curvature in sharp contrast to the negative curvature for quenching with $\mathrm{Co}^{2+}[12]$. When this behaviour is combined with the results of the time-resolved analysis there is good evidence that, although the Förster model provides a good decay-curve parameterisation, a different quenching mechanism is dominant for $\mathrm{Ni}^{2+}$. We thus investigated the behaviour of perylene fluorescence quenching by $\mathrm{Ni}^{2+}$ in glycerol, a solvent in which the Förster model had proved correct for $\mathrm{Co}^{2+}$ quenching[6]. Here again evidence was found for a different quenching mechanism other than Förster dipole-dipole energy transfer. In a plot of $\gamma$ vs $\left[\mathrm{Ni}^{2+}\right]$ the curves do not pass through zero which again places the application of the Förster model in doubt ${ }^{[12]}$.

Table 2 Kinetic parameters obtained from the analysis using 2-D Förster kinetics for the fluorescence quenching of perylene by $\mathrm{Ni}^{2+}$ ions in DPPC vesicles at $30^{\circ} \mathrm{C}$ and a lipid probe ratio of $358: 1$.

\begin{tabular}{cccc}
$\mathrm{Ni}^{2+}\left(\mathbf{m o l ~ I} \mathbf{l}^{-1}\right)$ & $\tau_{\mathbf{0}}(\mathbf{n s})$ & $\gamma$ & $\chi^{2}$ \\
\hline 0.033 & $6.66 \pm 0.06$ & 0.130 & 1.14 \\
0.068 & $6.44 \pm 0.07$ & 0.151 & 1.24 \\
0.106 & $6.31 \pm 0.07$ & 0.181 & 1.10 \\
0.137 & $6.17 \pm 0.08$ & 0.214 & 1.31 \\
0.174 & $6.00 \pm 0.07$ & 0.246 & 1.05 \\
0.209 & $5.95 \pm 0.07$ & 0.257 & 1.16 \\
0.253 & $5.79 \pm 0.07$ & 0.306 & 1.17 \\
0.285 & $5.79 \pm 0.07$ & 0.309 & 1.08 \\
0.321 & $5.56 \pm 0.07$ & 0.333 & 1.19 \\
0.353 & $5.61 \pm 0.07$ & 0.316 & \\
\hline
\end{tabular}


The evidence presented so far is strongly against the perylene-Ni ${ }^{2+}$ interaction being Förster type, without pointing towards an alternative source of quenching mechanism. At present the most likely quenching mechanism looks to be that of Dexter exchange interaction. The direct evidence for this is shown in Fig 4. Here $\phi / \phi_{0}$ and $\tau / \tau_{0}$ are plotted vs $\left[\mathrm{Ni}^{2+}\right.$. A fit to the $\phi / \phi_{0}$ data using the Förster mechanism (Eqn 4 ) is poor, whereas the exponential curvature predicted by the Dexter mechanism (Eqn 7) gives a much better fit over all the data. Further evidence for a Dexter mechanism in the case of $\mathrm{Ni}^{2+}$ is given by applying Eqn (8) to the data at the lower $\mathrm{Ni}^{2+}$ concentrations. In this case $\rho$ is found to be $\sim 0.13$ consistent with Dexter not Förster energy transfer.

The previous results demonstrate a potential method of fabricating "intelligent ion sensors" based on Förster energy transfer. The results in figure 2 clearly demonstrate donor-acceptor energy transfer across an interface and a simple relationship between $\gamma$ and $\left[\mathrm{Co}^{2+}\right]$. Such sensors could be based on Langmuir-Blodgett films, polymers, etc. The ability to tune the spectral properties of the donor to that of the acceptor should give the specificity required to analyse mixtures. Moreover, the "non-contact" nature of the Förster mechanism makes such a sensor independent of collisional quenching effects, eg. from oxygen. Further work in developing such sensors is in progress and will be reported shortly.

\section{Acknowledgement}

We wish to thank the SERC for financial support.

\section{REFERENCES}

1. J R Lakowicz, Principles of Fluorescence Spectroscopy, Plenum (1983).

2. J A Kemlo and T M Shepherd, Chem. Phys. Letts., 47, 158-162 (1977).

3. M J Aguirre, E A Lissi and A F Olea, J. Photochem., 36, 177-183 (1987).

4. M D Barkley, A Kowalczyk and L Brand, J. Chem. Phys., 75, 3581-3593 (1981).

5. J R Lakowicz and J R Knutson, Biochem, 19, 905-911 (1980).

6. T Salthammer, H Dreeskamp, D J S Birch and R E Imhof, J. Photochem. Photobiol. A:Chem, 55, 53-62 (1980).

7. A S Holmes, D J S Birch, K Suhling, R E Imhof, T Salthammer and H Dreeskamp, Chem. Phys. Letts., 186, 189-194 (1991).

8. D J S Birch and R E Imhof, Rev. Sci. Instrum., 52, 1206-1212 (1981).

9. For a recent review see $M$ Kaschke and $K$ Vogler, Laser Chem, 8, 19-38 (1988).

10. D L Huber, Phys. Rev. B, 20, 2307-2314 (1979).

11. V Breuninger and A Weller, Chem. Phys. Letts., 23, 40-44 (1973).

12. D J S Birch, K Suhling, A S Holmes, T Salthammer and R E Imhof, SPIE Proceedings, 1640, 707 (1992). 